\title{
Simulation of Collective Excitations in Long Josephson Junction Stacks
}

\author{
Ilhom Rahmonov ${ }^{1,2, \star}$, Yury Shukrinov ${ }^{1,3, \star \star}$, Pavlina Atanasova ${ }^{4}$, Elena Zemlyanaya ${ }^{3,5}$, \\ Oksana Streltsova ${ }^{5,6}$, Maxim Zuev ${ }^{5}$, Andrej Plecenik ${ }^{7}$, and Akinobu Irie ${ }^{8}$ \\ ${ }^{1}$ BLTP, Joint Institute for Nuclear Research, Dubna 141980, Russia \\ 2 Umarov Physical Technical Institute, Tajik Academy of Science, Dushanbe 734063, Tajikistan \\ ${ }^{3}$ Dubna State University, Dubna 141980, Russia \\ ${ }^{4}$ University of Plovdiv Paisii Hilendarski, Plovdiv 4000, Bulgaria \\ ${ }^{5}$ LIT, Joint Institute for Nuclear Research, Dubna 141980, Russia \\ ${ }^{6}$ Peoples' Friendship University of Russia (RUDN University), Moscow, Russia \\ ${ }^{7}$ Department of Experimental Physics, Comenius University, Bratislava, Slovakia \\ ${ }^{8}$ Utsunomiya University, Utsunomiya 321-8585, Japan
}

\begin{abstract}
The phase dynamics of a stack of long Josephson junctions has been studied. Both inductive and capacitive couplings between Josephson junctions have been taken into account in the calculations. The IV-curve, the dependence on the bias current of the radiation power and dynamics of each JJs of the stack have been investigated. The coexistence of the charge traveling wave and fluxon states has been observed. This state can be considered as a new collective excitation in the system of coupled Josephson junctions. We demonstrate that the observed collective excitation leads to the decrease of radiation power from the system.
\end{abstract}

\section{Introduction}

The layered high-Tc superconducting materials such as $\mathrm{Bi}_{2} \mathrm{Sr}_{2} \mathrm{CaCu}_{2} \mathrm{O}_{8+\delta}$ (BSCCO) can be considered as stacks of coupled Josephson junctions (JJs) [1]. The interest to the investigation of this system is caused by its rich nonlinear properties and different applications, particularly this system is one of the promising object of superconducting electronics [2]. The JJs stack demonstrates a series of interesting properties such as parametric resonance [3-5], chaotic features [6], and the occurrence of the fluxons [7-9] and collective excitations [10, 11]. Coherent terahertz electromagnetic radiation from this system provides wide possibilities for various applications $[12,13]$. The effect of collective excitations on radiation power from the stack is not studied in detail yet. In this paper, we investigate the IV-curve of stacked JJs and radiation power from the stack taking into account the inductive and capacitive couplings $[14,15]$ and the diffusion current $[5,16,17]$.

The system of equations which describes the phase dynamics of the coupled long JJs stack in the normalized quantities can be written as follows [14, 15, 18].

\footnotetext{
^e-mail: rahmonov@theor.jinr.ru

$\star \star$ e-mail: shukrinv@ theor.jinr.ru
} 


$$
\left\{\begin{array}{l}
\frac{\partial \varphi_{l}}{\partial t}=D_{c} V_{l}+s_{c} V_{l+1}+s_{c} V_{l-1} \\
\frac{\partial V_{l}}{\partial t}=\sum_{k=1}^{N} £_{l k}^{-1} \frac{\partial^{2} \varphi_{k}}{\partial x^{2}}-\sin \varphi_{l}+\beta \frac{\partial \varphi_{l}}{\partial t}+I
\end{array}\right.
$$

where $\varphi_{l}$ and $V_{l}$ are the phase difference and voltage of $l$ th $\mathrm{JJ}$, respectively. $I$ is the bias current normalized to the critical current $j_{c}, \beta$ is a dissipation parameter, $D_{c}=1+\left(2 \lambda_{e} / d_{I}\right) \operatorname{coth}\left(d_{s} / \lambda_{e}\right)$ is the effective electrical thickness of JJ normalized to the insulating layer thickness $d_{I}, s_{c}=-\lambda_{e} /\left[d_{I} \sinh \left(d_{s} / \lambda_{e}\right)\right]$ is the capacitive coupling parameter, $d_{s}$ is the thickness of the superconducting layer, and $\lambda_{e}$ is Debye screening length. The voltage is normalized to $V_{0}=\hbar \omega_{p} /(2 e)$, where $\omega_{p}=\sqrt{8 \pi d_{I} e j_{c} /(\hbar \varepsilon)}$ is the plasma frequency of $\mathrm{JJ}$, and $\varepsilon$ is the dielectric constant of the insulating layer. The time $t$ and coordinate $x$ are normalized to the plasma frequency $\omega_{p}^{-1}$ and the Josephson penetration depth $\lambda_{J}$, respectively. The matrix of inductive coupling $\hat{f}$ has the form

$$
\hat{\mathfrak{E}}=\left(\begin{array}{ccccccc}
1 & S & 0 & \cdots & & 0 & S \\
S & 1 & S & 0 & & & 0 \\
& \ddots & \ddots & \ddots & \ddots & & \\
\cdots & 0 & S & 1 & S & 0 & \cdots \\
& & \ddots & \ddots & \ddots & \ddots & \\
0 & & & 0 & S & 1 & S \\
S & 0 & & & 0 & S & 1
\end{array}\right) \text {, }
$$

where $S=s_{£} / D_{£}$ is the inductive coupling parameter, $s_{\mathfrak{1}}=-\lambda_{L} / \sinh \left(d_{s} / \lambda_{L}\right), D_{£}=d_{I}+$ $2 \lambda_{L} \operatorname{coth}\left(d_{s} / \lambda_{L}\right)$ is the effective magnetic thickness of $\mathrm{JJ}$, and $\lambda_{L}$ is the London penetration depth. Valid values of the inductive coupling parameter $S$ are in the range $S \in(-0.5,0)$. The initial conditions for the system of equations $(1)$ are $\varphi_{l}(x, 0)=0$ and $V_{l}(x, 0)=0$. The boundary conditions in the $x$ dirrection are given by the external magnetic field $\partial \varphi_{l} /\left.\partial x\right|_{x=0, L}=B_{\text {ext }}$. The magnetic field is normalized to $B_{0}=\hbar c / 2 e D_{£}$. In the $z$ direction we use periodic boundary conditions: at $l=N$, $\varphi_{l+1}=\varphi_{1}, V_{l+1}=V_{1} ;$ at $l=1, \varphi_{l-1}=\varphi_{N}, V_{l-1}=V_{N}$.

Simulations are based on the numerical solution of the system (1) by the fourth order RungeKutta method, the finite-difference approximation of the spatial derivatives, and the MPI technique for parallel implementation. The details of simulation of the IV-curve and other characteristics are discussed in our previous papers $[5,18,19]$. The power of radiation from the stack is simulated using the expression $P=V_{\mathrm{AC}}^{2} / R_{Z}[18,20]$, where $V_{\mathrm{AC}}=d_{I} E_{\mathrm{AC}}, E_{\mathrm{AC}}$ is the AC part of the electric field, $R_{Z}=\left(d_{I} / W\right) Z, W$ is the width of $\mathrm{JJ}, Z$ is the impedance of the radiation. Details of simulation of the radiation power are discussed in Ref. [18, 20]. The magnetic field in the JJs is calculated using the expression $B_{l}=B_{0} \sum_{k=1}^{N} £_{l k}^{-1} \partial \varphi_{k} / \partial x$. The charge in superconducting layers is calculated using the expression $Q_{l}(x, t)=Q_{0}\left[V_{l}(x, t)-V_{l-1}(x, t)\right][5]$, where $Q_{0}=\varepsilon V_{0} / 4 \pi d_{s} d_{I}$.

\section{Results and discussion}

In Fig. 1 the IV-curve and power of radiation as a function of bias current for the stack of ten JJs are presented. Calculation is provided for a stack with dissipation parameter $\beta=0.2$, capacitive coupling $s_{c}=-0.05$ and effective electric thickness $D_{c}=1.1$. The typical BSCCO parameter $S$ is close to -0.5 [21]. However, in order to clarify the influence of the inductive coupling we choose the weak coupling parameter $S=-0.05$. The IV-curve demonstrates seven zero field steps (ZFS) [7-9], which are formed due to the appearance of fluxons (kinks and antikinks). The vertical dashed lines mark the boundaries of the regions of IV-curve where fluxons appear. For each region the corresponding number of fluxons is indicated. Between the regions of the IV-curve with one and two fluxons near 
some ZFSs in comparison with the case of single $\mathrm{JJ}$ there appears an additional branch due to the different number of fluxons in each JJs of the stack. This feature was discussed in [18]. In the case of single JJ a significant radiation power is observed in the regions of IV-curve corresponding to the ZFS [18]. In the case of the stacked JJs the radiation power is close to zero near some ZFSs in comparison with the case of a single JJ (in our case ZFS with 5 and 7 fluxons).

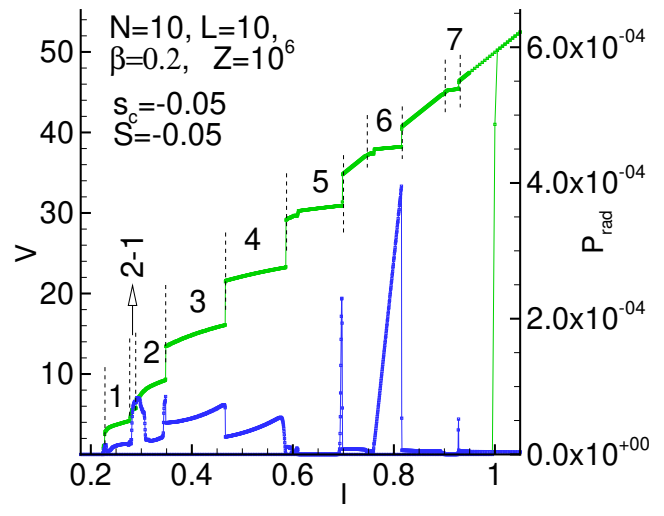

(a)

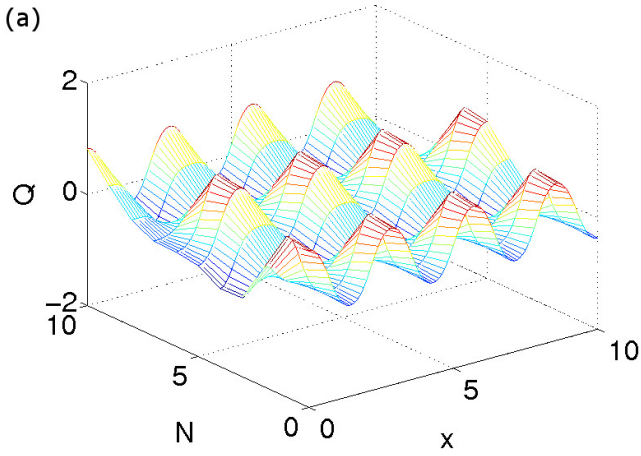

Figure 1. IV-curve together with bias current dependence of the radiation power calculated for a stack of $N=10 \mathrm{JJs}$ with model parameters $D_{c}=1.1, s_{c}=-0.05, S=-0.05$ and $\beta=0.2$. Vertical dashed lines show the boundaries of ZFS. For each ZFS the number of fluxons is indicated.

Figure 2. (a) Charge distribution in superconducting layers along the coordinate and stack of JJs at $I=0.92$ at a fixed time moment; (b) Distribution of phase difference (solid line) and magnetic field (doted line) along the coordinate at $I=0.92$ at a fixed time moment for first JJ. The boundaries of fluxons and antifluxons are shown with the vertical dashed line; (c) The same as in the case (b) for fifth JJ.

In order to explain such behavior of stacked JJs we have investigated the dynamics of each JJs of the stack and calculated the charge dynamics in superconducting layers in the above mentioned region of the IV-curve. Figure 2(a) demonstrates the distribution of charge in superconducting layers along the stack and coordinate, for fixed time moment at $I=0.92$. It can be seen that along the stack a wave-like behavior of the charge is realized. Along the coordinate we observe the fluxons. The analysis of the dynamics of each JJs of the stack demonstrates that along the stack a charge traveling wave (CTW) appears, i.e. the CTW and fluxons coexist. Such dynamical state can be considered as a new collective excitation in the stacked JJs.

The question arises why the observed collective excitation leads to the decrease of the radiation power. In order to explain this phenomena we have analyzed the dynamics of the phase difference and magnetic field for each JJs of the stack. In Fig. 2(b) the spatial distribution of the phase difference (solid line) and magnetic field (doted line) at the fixed time moment in the first JJ at the current value 
$I=0.92$ are shown, which corresponds to the seventh ZFS. This dependence demonstrates seven fluxons (four fluxons and three antifluxons). The same characteristics for the middle of stack, i.e., in the fifth JJ are presented in Fig. 2(c). In comparison with the first JJ (Fig. 2(b)), here we can see four antifluxons and three fluxons, i.e., the opposite situation. This lets us conclude that the first and fifth JJs are of opposite phases. This circumstance results in the zero value of the average radiation power.

\section{Conclusions}

In this paper we have investigated the structure of the IV-curve of JJs and the radiation power emitted by a stack of JJs. We demonstrate that in the stack of JJs a charge traveling wave can appear in the ZFS, i.e. fluxons coexist with the charge traveling wave. This points to the appearance of a new collective excitation in the system of coupled JJs. We note that such collective excitation in the stack of JJs has a significant influence on the magnitude of the radiation power from the stack of JJs. Particularly, it leads to a zero radiation power from the stack. The obtained results are of interest to the analysis of the experimental IV-curves.

\section{Acknowledgement}

The study was funded by the RFBR, according to the research project 15-29-01217, HeisenbergLandau program, JINR-Slovakia collaboration, JINR-Bulgaria cooperation program, project FP17FMI-008, and by the Ministry of Education and Science of the Russian Federation (the Agreement number 02.a03.21.0008).

\section{References}

[1] R. Kleiner, F. Steinmeyer, G. Kunkel, and P. Müller, Phys. Rev. Lett. 68, 2394 (1992)

[2] A.A. Yurgens, Supercond. Sci. Technol. 13, R85 (2000)

[3] Yu. M. Shukrinov and F. Mahfouzi, Phys. Rev. Lett. 98, 157001 (2007)

[4] Yu. M. Shukrinov, F. Mahfouzi, and M. Suzuki, Phys. Rev. B 78, 134521 (2008)

[5] I. R. Rahmonov, Y. M. Shukrinov, and A. Irie, JETP Letters 99, 632 (2014)

[6] Yu. M. Shukrinov, A. E. Botha, S. Yu. Medvedeva, M. R. Kolahchi, and A. Irie, Chaos 24, 033115 (2014)

[7] T. A. Fulton and R. C. Dynes, Solid St. Commun. 1257 (1972)

[8] N. F. Pedersen and D. Welner, Phys. Rev. B 29, 2551 (1984)

[9] D. W. McLaughlin and A. C. Scott, Phys. Rev. A 18, 1652 (1978)

[10] R. Kleiner, T. Gaber, and G. Hechtfischer, Phys. Rev. B 62, 4086 (2000)

[11] Y. Matsuda, M.B. Gaifullin, K. Kumagai, K. Kadowaki, and T. Mochiku, Phys. Rev. Lett. 75, $4512(1995)$

[12] L. Ozyuzer, A.E. Koshelev, C. Kurter et al., Science 318, 1291 (2007)

[13] U. Welp, K. Kadowaki and R. Kleiner, Nature Photonics 7, 702 (2013)

[14] S. Sakai, P. Bodin, and N.F. Pedersen, J. Appl. Phys. 73, 2411 (1993)

[15] M. Machida and S. Sakai, Phys. Rev. B 70, 144520 (2004)

[16] Y. M. Shukrinov and F. Mahfouzi, Physica C 434, 6 (2006)

[17] Y. M. Shukrinov and I. R. Rahmonov, JETP 115, 289 (2012)

[18] I. R. Rahmonov, Yu. M. Shukrinov, P. Kh. Atanasova, E. V. Zemlyanaya, and M. V. Bashashin, JETP 124131 (2017)

[19] Yu. Shukrinov, I. Rahmonov, and M. Hamdipour, LNCS 7125, 234 (2012)

[20] V. M. Krasnov, Phys. Rev. B 82, 134524 (2010)

[21] V. M. Krasnov, Phys. Rev. B, 83, 174517, (2011) 